\title{
The responses of Aboriginal Canadians to adjuvanted pandemic (H1N1) 2009 influenza vaccine
}

\author{
Ethan Rubinstein MD, Gerald Predy MD, Laura Sauvé MD, Greg W. Hammond MD, Fred Aoki MD, \\ Chris Sikora MD, Yan Li PhD, Barbara Law MD, Scott Halperin MD, David Scheifele MD
}

\begin{abstract}
Background: Because many Aboriginal Canadians had severe cases of pandemic (H1N1) 2009 influenza, they were given priority access to vaccine. However, it was not known if the single recommended dose would adequately protect people at high risk, prompting our study to assess responses to the vaccine among Aboriginal Canadians.
\end{abstract}

Methods: We enrolled First Nations and Métis adults aged 20-59 years in our prospective cohort study. Participants were given one $0.5-\mathrm{mL}$ dose of ASO3-adjuvanted pandemic (H1N1) 2009 vaccine (Arepanrix, GlaxoSmithKline (anada). Blood samples were taken at baseline and 21-28 days after vaccination. Paired sera were tested for hemagglutinationinhibiting antibodies at a reference laboratory. To assess vaccine safety, we monitored the injection site symptoms of each participant for seven days. We also monitored patients for general symptoms within 7 days of vaccination and any use of the health care system for 21-28 days after vaccination.

Results: We enrolled 138 participants in the study (95 First Nations, 43 Métis), 137 of whom provided all safety data and 136 of whom pro- vided both blood samples. First Nations and Métis participants had similar characteristics, including high rates of chronic health conditions $(74.4 \%-76.8 \%)$. Pre-existing antibody to the virus was detected in $34.3 \%$ of the participants, all of whom boosted strongly with vaccination (seroprotection rate [titre $\geq 40$ ] $100 \%$, geometric mean titre 531-667). Particpants with no pre-existing antibody also responded well. Fifty-eight of 59 (98.3\%) First Nations participants showed seroprotection and a geometric mean titre of 353.6; all 30 Métis participants with no pre-existing antibody showed seroprotection and a geometric mean titre of 376.2. Pain at the injection site and general symptoms frequently occurred but were shortlived and generally not severe, although three participants $(2.2 \%)$ sought medical attention for general symptoms.

Interpretation: First Nations and Métis adults responded robustly to ASO3-adjuvanted pandemic (H1N1) 2009 vaccine. Virtually all participants showed protective titres, including those with chronic health conditions.

Trial registration: ClinicalTrials.gov trial register no. NCT.01001026.
Competing interests: David Scheifele, Scott Halperin, Gerald Predy, Chris Sikora and Fred Aoki have conducted previous influenza vaccine studies with research funds from vaccine manufacturers GlaxoSmithKline and Sanofi Pasteur. No other competing interests were declared.

This article has been peer reviewed.

Correspondence to: Dr. David Scheifele, dscheifele@cfri.ca

CMAJ 2011. DOI:10.1503 /cmaj.110196
$\mathrm{D}$ uring the first wave of the H1N1 pandemic in Canada in 2009, some First Nations communities were severely affected, with younger adults and children most at risk for severe disease. ${ }^{1,2}$ Whereas Aboriginal Canadians make up $3.4 \%$ of the population (with 1.14 million people), they accounted for $16 \%$ of admissions to hospital during the first wave of the pandemic, and $43 \%$ of Aboriginal patients had underlying medical conditions. ${ }^{3}$ The increased rate of severe disease might have resulted from residential crowding, prevalence of chronic health conditions, delayed access to health care or suboptimal immune responses to infection. ${ }^{4}$ When a federally funded, ASO3adjuvanted (squalene/tocopherol) pandemic vaccine became available for Canadians later in 2009,5 Aboriginal people were given priority access to it. ${ }^{3}$ However, dosing requirements at the time were tentative. Previous studies of an ASO3-adjuvanted influenza A (H5N1) vaccine established that two doses were needed for im munity in adults. ${ }^{6}$ Because the 2009 influenza (H1N1) pandemic occurred without warning, no prepandemic studies had been done with vaccines based on this novel swine-derived virus. $^{\text {? }}$

The ASO3-adjuvanted pandemic (H1N1) 2009 vaccine manufactured in Canada (Arepanrix, GlaxoSmithKline, Laval, Quebec) was released for public use as soon as it was available, unstudied, to mitigate morbidity during the pandemic's second wave, which was already in progress. A single 3.75- $\mu \mathrm{g}$ dose of hemagglutinin was recommended for adults using the preliminary results of a European trial of another 
ASO3-adjuvanted vaccine (Pandemrix, GlaxoSmithKline, Rixensart, Belgium) given to 65 adults aged $18-60$ years. ${ }^{8}$ The European product was believed to be equivalent to the Canadianmade vaccine, but this had not yet been shown.

We wondered if the recommended single dose would be adequate for Aboriginal Canadian adults given their heightened risk of severe influenza during the first wave. We were unable to identify any previous studies of influenza vaccines involving Aboriginal Canadians to determine if their responses would be similar to other Canadians or to the healthy European study participants on whom the dosing recommendation was based. Consequently, we undertook a study involving First Nations and Métis adults to assess their responses to the pandemic vaccine.

\section{Methods}

Eligible participants were self-identified First Nations or Métis adults aged 20-59 years, including people with chronic health conditions not associated with immune dysfunction. Most of the participants recruited to the study were urban residents. Exclusion criteria included pregnancy, allergy to eggs, bleeding disorders and prior receipt of the 2009-10 seasonal trivalent influenza vaccine. The study period lasted from November 2009 to January 2010 during the second wave of the influenza pandemic in Canada.

Blood samples to measure titres of hemagglutination-inhibiting antibodies against the pandemic (H1N1) 2009 influenza virus were obtained at baseline and 21-28 days after vaccination. Participants were given a single $0.5-\mathrm{mL}$ dose of Arepanrix vaccine injected into the deltoid muscle. One commercial lot of vaccine was used containing $3.75 \mu \mathrm{g}$ (H1N1) 2009 influenza hemagglutinin per dose, with $\mathrm{ASO} 3$ adjuvant. ${ }^{5}$ To assess vaccine safety, participants kept a daily diary for seven days after vaccination to record any changes at the injection site or general symptoms, including fever. Thereafter, only health events requiring medical attention were documented.

At the first visit, participants were given the vaccination and a nasal test kit (flocked swab, transport medium) to take home and use if they developed an influenza-like illness (with cough and fever) while enrolled in the study. Participants were provided with instructions for using the kit, and reminders were given during each subsequent contact. When a kit was used, arrangements were made to collect the sample and have it tested for influenza virus by polymerase chain reaction at a central laboratory.

Study staff reviewed the diaries and influenzalike illnesses with participants by telephone on day 7 and in person 21-28 days after vaccination. Paired sera were tested for hemagglutinationinhibiting antibodies at the National Microbiology Laboratory in Winnipeg, Manitoba. Standard criteria were used to assess antibody responses, ${ }^{9}$ including seroprotection rates, geometric mean titres, geometric mean fold rises and seroconversion rates. Antibody titres were expressed as the reciprocal value (e.g., 40 in place of 1:40). Because protocol adherence was excellent, the same participants were included in the immunogenicity and safety analyses.

We had intended to enrol 200 participants in the study. If a minimum of 110 evaluable participants could be enrolled, the study would have at least $80 \%$ power to detect a $15 \%$ or greater departure from the $100 \%$ seroprotection rate reported in the earlier study of ASO3-adjuvanted pandemic (H1N1) 2009 influenza vaccine. ${ }^{8}$

Fisher exact test was used to compare proportional data, and analysis of variance (ANOVA) was used to compare continuous data, including geometric mean titre and geometric mean fold rise.

This study was undertaken by the Public Health Agency of Canada/Canadian Institutes of Health Research Influenza Research Network (PCIRN) centres in Winnipeg; Edmonton, Alberta; and Vancouver, British Columbia. The funders played no role in the analysis of the data or in the design and conduct of the study. Aboriginal community leaders were consulted to obtain their approval and advice for implementing the protocol in a culturally appropriate manner. The protocol was approved by the research ethics boards of Health Canada and the participating institutions, including the University of British Columbia Clinical Research Ethics Board as the lead institution. The trial was registered with ClinicalTrials.gov as trial number NCT.01001026. Written informed consent was obtained from each participant upon enrollment in the study.

\section{Results}

We enrolled 138 participants in the study (100 from Winnipeg, 27 from Edmonton and 11 from Vancouver), and 137 participants provided symptom summaries at both interviews. Blood samples were obtained from 137 participants at baseline and from 136 participants after vaccination, for a protocol completion rate of $98.6 \%$.

The demographics of the participants from the First Nations $(n=95)$ and Métis $(n=43)$ groups were similar (Table 1). First Nations participants gave $25 \mathrm{Nation} / \mathrm{Band}$ affiliations, with Ojibway (33 people) and Cree (23 people) predominating. Underlying health conditions were reported by $76.8 \%$ of First Nations participants 
and by $74.4 \%$ of Métis participants. The most commonly reported conditions were respiratory (17 participants, 8 of whom reported asthma), cardiovascular (17 participants, 13 of whom reported hypertension), endocrine (15 participants, 8 of whom reported diabetes mellitus and 7 of whom reported thyroid disorders), gastrointestinal (12 participants, 3 of whom reported chronic hepatitis), dermatologic (12 participants, 6 of whom reported eczema and 3 of whom reported psoriasis), psychological (11 participants, 10 of whom reported depression) and hematologic disorders (7 participants, 6 of whom reported anemia). Eleven participants had body mass indices greater than $40 \mathrm{~kg} / \mathrm{m}^{2}$. Thirty-eight participants (27.5\%) had conditions that posed high risk for influenza complications as per Canadian guidelines. ${ }^{10}$ None of the participants were receiving immunosuppressive medications, including one participant with sarcoidosis and one participant with rheumatoid arthritis.

Hemagglutination-inhibiting antibody responses are summarized in Table 2. Pre-existing antibody to the pandemic (H1N1) 2009 influenza virus was detectable in about one-third of participants $(47,34.3 \%)$. The responses of primed (baseline titre $\geq 10$ ) and naive participants (i.e., people with no detectable antibody at baseline) were assessed separately. After vaccination, at least $99.3 \%$ (95\% confidence interval [CI] 96.0100.0) of First Nations and Métis participants had protective titres $(\geq 40)$. Primed and naive participants in both groups mounted robust responses as measured by geometric mean titres (Table 2). However, postvaccination geometric mean titres were significantly higher in primed participants than in naive participants (ANOVA, $p<0.01$ ).

Symptoms during the week after vaccination were frequent but generally mild (Appendix 1, available at www.cmaj.ca/lookup/suppl/doi:10 $.1503 / \mathrm{cmaj} .110196 /-/ \mathrm{DC} 1)$. Pain at the injection site was noted by $75.9 \%$ of participants, but only $5.8 \%$ rated it as being severe (i.e., limiting the participant's activities). Redness at the injections site was reported by $12.4 \%$ of participants, and swelling was reported by $18.2 \%$ of participants. The reported rates of general symptoms during the week after vaccination were as follows: myalgia, $54.0 \%$; tiredness, $52.6 \%$; headache, $40.1 \%$; malaise, $32.8 \%$; and arthralgia, $26.3 \%$. No fevers of $38.0^{\circ} \mathrm{C}$ or higher were recorded. Most symptoms resolved by day 6 postvaccination. Three participants (2.2\%) sought medical attention for general symptoms, and no one sought medical attention for local symptoms. Three participants required admission to hospital during the study period for illnesses unrelated to vaccination. Three participants described having an influenza-like illness before their final visit, but the results of polymerase chain reaction tests for influenza were negative.

\section{Interpretation}

Both First Nations and Métis adults responded robustly to the adjuvanted vaccine. Participants with evidence of prior infection with the pandemic (H1N1) 2009 influenza virus boosted well with vaccination (Table 2 ), and those with no pre-existing antibodies also had strong responses to vaccination. The observed seroprotection rate of 98.8\% (95\% CI 94.2-100.0) among naive participants was remarkable, exceeding the $89.2 \%$ (95\% CI 81.1-94.7) seroprotection rate of naive, non-Aboriginal adults in a concurrent PCIRN study of the same vaccine involving people in the same age group. ${ }^{11}$

The sizes of response (geometric mean titres) were significantly higher among First Nations and Métis participants (Table 2) than among non-Aboriginal Canadian adults (geometric mean titre $141,95 \%$ CI 109-183) in the concurrent study, ${ }^{11}$ attesting to the adequacy of responses of both First Nations and Métis participants. However, the "seroprotection rate" is a correlate of protection based on seasonal influenza vaccines, which might not apply to a pandemic virus. With seasonal vaccines, a hemagglutination-inhibiting antibody titre of $1: 40$ in young adults correlated to $50 \%$ protective efficacy, with higher titres affording greater protection. ${ }^{12}$ The mean titre in the current study was 10-fold higher than the threshold value, thus

Table 1: Characteristics of the 138 First Nations and Métis adults enrolled in the study

\begin{tabular}{|c|c|c|c|}
\hline \multirow[b]{2}{*}{ Characteristic } & \multicolumn{2}{|c|}{ No. $(\%)^{*}$} & \multirow[b]{2}{*}{$p$ value $\dagger$} \\
\hline & $\begin{array}{l}\text { First Nations } \\
\quad n=95\end{array}$ & $\begin{array}{l}\text { Métis } \\
n=43\end{array}$ & \\
\hline Age, yr, mean (SD) & $36.2(10.1)$ & $39.7(12.2)$ & $0.08 \ddagger$ \\
\hline \multicolumn{4}{|l|}{ Sex } \\
\hline Men & $34(35.8)$ & $13(30.2)$ & $0.57 \S$ \\
\hline Women & $61(64.2)$ & $30(69.8)$ & - \\
\hline $\begin{array}{l}\text { Body mass index, } \\
\mathrm{kg} / \mathrm{m}^{2} \text {, mean (SD) }\end{array}$ & $29.6 \quad(5.9)$ & $30.2 \quad(6.5)$ & $0.58 \ddagger$ \\
\hline $\begin{array}{l}\text { One or more chronic } \\
\text { health conditions }\end{array}$ & $73(76.8)$ & $32(74.4)$ & $0.83 \S$ \\
\hline $\begin{array}{l}\text { Past influenza } \\
\text { vaccination }\end{array}$ & $46(48.4)$ & $14(32.6)$ & $0.10 \S$ \\
\hline \multicolumn{4}{|c|}{$\begin{array}{l}\text { Note: SD = standard deviation. } \\
\text { *Unless otherwise indicated. } \\
\text { †No statistically significant differences existed between groups. } \\
\text { ¥Analysis of variance. } \\
\text { §Fisher exact test. }\end{array}$} \\
\hline
\end{tabular}


increasing the likelihood of protection.

The robust nature of the immune response of Aboriginal adults to influenza vaccine was unanticipated. An analogous situation might be hepatitis C infection, which North American Aboriginal people have an enhanced capacity to resolve. ${ }^{13}$ Studies of innate responses of First Nations volunteers in Manitoba ${ }^{14}$ showed a greater tendency toward a proinflammatory cytokine milieu in response to hepatitis $\mathrm{C}$ virus, potentially aiding clearance of infection. A similar proinflammatory innate response to adjuvanted influenza vaccine could plausibly result in stronger antibody responses. A greater pro-inflammatory response to influenza infection might be the basis for the severe disease that affected some First Nations people during the pandemic. ${ }^{1,2}$

Aboriginal participants reported having adverse events during the week after immunization at rates similar to those seen in other studies that evaluated ASO3-adjuvanted pandemic (H1N1)
2009 vaccines in adults. ${ }^{5,15}$ No significant differences in these rates were seen between the Aboriginal participants in our study and the nonAboriginal participants in the concurrent PCIRN study. ${ }^{11}$ Pain at the injection site and general symptoms of tiredness or myalgia were described by over half of the participants in this study during the week after vaccination. These and other symptoms were generally mild and short-lived, having little impact on the ability to perform daily activities. No serious adverse events were attributed to vaccination.

\section{Limitations}

Participant selection is a challenge for studies involving Aboriginal Canadians, given the large number of First Nations, their differing ethnography and their often remote locations. We believe that a strength of our study was recruitment in cities with substantial Aboriginal populations, enabling the selection of participants from

Table 2: Hemagglutination-inhibiting antibody responses to adjuvanted pandemic (H1N1) 2009 influenza vaccine among First Nations and Métis adults enrolled in the study

\begin{tabular}{|c|c|c|}
\hline \multirow[b]{2}{*}{ Antibody response } & \multicolumn{2}{|c|}{ No. $(\%)^{*}$} \\
\hline & $\begin{array}{l}\text { First Nations } \\
\quad n=94\end{array}$ & $\begin{array}{l}\text { Métis } \\
n=43\end{array}$ \\
\hline \multicolumn{3}{|l|}{ Baseline } \\
\hline Titre $\geq 10$ & $34 \quad(36.2)$ & $13(30.2)$ \\
\hline Seroprotective titre $\geq 40$ & $16(17.0)$ & $7(16.3)$ \\
\hline Geometric mean titre $(95 \% \mathrm{Cl})$ & $10.1 \quad(8.0-12.8)$ & $8.6 \quad(6.4-11.7)$ \\
\hline \multicolumn{3}{|l|}{ Postvaccination } \\
\hline Seroconversion $\geq 4$-fold titre increase & $\begin{array}{c}n=93 \\
86(92.5)\end{array}$ & $\begin{array}{c}n=43 \\
42 \quad(97.7)\end{array}$ \\
\hline Naive & $\begin{array}{c}n=59 \\
58(98.3) \dagger\end{array}$ & $\begin{array}{c}n=30 \\
30(100.0)\end{array}$ \\
\hline Primed & $\begin{array}{c}n=34 \\
28(82.4) \dagger\end{array}$ & $\begin{array}{c}n=13 \\
12 \quad(92.3)\end{array}$ \\
\hline Seroprotective titre $\geq 40$, no. $(\% ; 95 \% \mathrm{Cl})$ & $\begin{array}{c}n=93 \\
92 \quad(98.9 ; 94.2-100.0)\end{array}$ & $\begin{array}{c}n=43 \\
43(100.0 ; 91.8-100.0)\end{array}$ \\
\hline Naive & $\begin{array}{cc} & n=59 \\
58 & (98.3 ; 90.9-100.0)\end{array}$ & $\begin{array}{c}n=30 \\
30(100.0 ; 88.4-100.0)\end{array}$ \\
\hline Primed & $\begin{array}{c}n=34 \\
34(100.0 ; 89.7-100.0)\end{array}$ & $\begin{array}{c}n=13 \\
13(100.0 ; 75.3-100.0)\end{array}$ \\
\hline Geometric mean titre $(95 \% \mathrm{Cl})$ & $445.8(349.3-569.1)$ & $417.5(288.6-604.0)$ \\
\hline Naive & $353.6(261.9-477.3) \dagger$ & $376.2(234.1-604.4)$ \\
\hline Primed & $666.6(446.3-995.8) \dagger$ & $531.0(285.6-987.4)$ \\
\hline Geometric mean fold rise $(95 \% \mathrm{Cl})$ & $43.8 \quad(32.1-59.6)$ & $48.3 \quad(30.6-76.2)$ \\
\hline Naive & $69.5 \quad(51.5-93.8) \dagger$ & $73.5 \quad(45.9-117.8) \dagger$ \\
\hline Primed & $19.6 \quad(10.8-35.6) \dagger$ & $18.3 \quad(7.2-46.6) \dagger$ \\
\hline \multicolumn{3}{|c|}{$\begin{array}{l}\text { Note: } \mathrm{Cl}=\text { confidence interval. } \\
* \text { Unless otherwise indicated. } \\
t p<0.01 \text {, comparing primed (titre } \geq 10 \text { at baseline) and naive subjects. No significant differences existed between responses of } \\
\text { First Nations and Métis subjects. Seroconversion rates were compared using Fisher exact test, and geometric mean titre and } \\
\text { geometric mean fold rise were compared using analysis of variance. }\end{array}$} \\
\hline
\end{tabular}


25 different bands and First Nations, as well as Métis. Studying selected reserve communities would not have resulted in as broad a sample population. One-third of the participants had evidence of prior infection with pandemic (H1N1) 2009 influenza upon entry into the study, confirming that participants were drawn from populations at risk of exposure during the first wave. Including participants with chronic health conditions made the study more representative of the Aboriginal population, in contrast to typical vaccine studies that limit enrolment to healthy volunteers.

Because many potential participants had received the pandemic vaccine before our study began, our sample was smaller than intended but still within the desired range. Our results might not apply to all First Nations and Métis people, nor might they apply to Inuit adults, who were not included in the study but who had high rates of severe infection during the pandemic. ${ }^{3}$ Antibody titres might have been increased by subsequent exposures to the pandemic virus as the study was conducted during the second wave of infections. However, most participants were enrolled in Winnipeg, which had a milder second wave than the other sites.

There was a high prevalence of underlying medical conditions among the Aboriginal adults in this study. Many of these conditions pose an increased risk for severe influenza infection thus warranting annual vaccination. It would seem prudent to determine if satisfactory responses also follow the administration of unadjuvanted seasonal influenza vaccines among people at high risk.

\section{Conclusion}

Adjuvanted pandemic (H1N1) 2009 influenza vaccine resulted in high antibody titres in vulnerable First Nations and Métis people.

\section{References}

1. Zarychanski R, Stuart T, Kumar A, et al. Correlates of severe disease in patients with 2009 pandemic influenza (H1N1) virus infection. CMAJ 2010;182:257-64.

2. Kumar A, Zarychanski R, Pinto R, et al for the Canadian Critical Care Trials Group H1N1 Collaborative. Critically ill patients with 2009 influenza $\mathrm{A}(\mathrm{H} 1 \mathrm{~N} 1)$ infection in Canada. JAMA 2009; 302:1872-9.

3. Frequently asked questions - H1N1 flu virus. H1N1 in Aboriginal, First Nation and Inuit communities. Ottawa (ON): Public Health Agency of Canada; 2009. Available: www.phac-aspc .gc.ca/alert-alerte/h1n1/faq/faq_rg_h1n1-anic-eng.php. (accessed 2011 Jan. 20).

4. Deaths related to the 2009 pandemic influenza A (H1N1) among American Indian/Alaska Natives - 12 states, 2009. MMWR Morb Mortal Wkly Rep 2009;58:1341-4.

5. Roman F, Vaman T, Kafeja F, et al. ASO $3_{\mathrm{A}}$-adjuvanted in fluenza A (H1N1) vaccine for adults up to 85 years of age. Clin Infect Dis 2010;51:668-77.

6. Langley JM, Frenette L, Ferguson L, et al. Safety and cross- reactive immunogenicity of candidate ASO3-adjuvanted prepandemic H5N1 influenza vaccines: a randomized controlled phase 1/2 trial in adults. $J$ Infect Dis 2010;201:1644-53.

7. Smith GJ, Vijaykrishna D, Bahl J, et al. Origins and evolutionary genomics of the 2009 swine-origin H1N1 influenza A epidemic. Nature 2009;459:1122-5.

8. Pandemic (H1N1) 2009 influenza update: Results from second clinical trial of GSK's H1N1 adjuvanted vaccine confirm immune response and tolerability [press release]. London (UK): GlaxoSmithKline; 2009. Available: www.gsk.com/media /pressreleases/2009/2009_pressrelease_10111.htm (accessed 2009 Oct. 21).

9. Committee for Proprietary Medicinal Products (CHMP). Note for guidance on harmonization of the requirements for influenza vaccine. London (UK): The European Agency for the Evaluation of Medicinal Products; 1997. Available: www.ema.europa .eu/pdfs/human/bwp/021496en.pdf (accessed 2011 June 9).

10. Statement on influenza vaccination for the 2008-2009 season: an Advisory Committee Statement (ACS). Can Commun Dis Rep 2008;34:(ACS-3): 1-46.

11. Scheifele DW, Dionne M, De Serres G, et al. PCIRN study of adults given monovalent, adjuvated $\mathrm{H} 1 \mathrm{~N} 1$ vaccine with concurrent or delayed trivalent seasonal influenza vaccination. Canadian Immunization Conference, Quebec City, December 3-5, 2010 (abstract P141). Can J Infect Dis Med Microbiol 2010;21:226.

12. Hobson D, Curry RL, Beare AS, et al. The role of serum haemagglutination-inhibiting antibody in protection against challenge infection with influenza A2 and B viruses. $J$ Hyg (Lond) 1972;70:767-77.

13. Minuk GY, Uhanova J. Viral hepatitis in the Canadian Inuit and First Nations populations. Can J Gastroenterol 2003;17: 707-12.

14. Rempel JD, Aborsangaya KB, Alphonse MP, et al. The influence of North American Aboriginal ethnicity on pro-inflammatory and anti-inflammatory cytokine responses to IFN-alpha. J Viral Hepat 2009;16:292-7.

15. Mayet A, Ligier C, Gache K, et al. Adverse events following pandemic influenza vaccine Pandemrix reported in the French military forces - 2009-2010. Vaccine 2011;29:2576-81.

Affiliations: From the Health Sciences Center, University of Manitoba (Rubinstein, Hammond, Aoki) Winnipeg, Man.; Alberta Health Services (Predy, Sikora), Edmonton, Alta.; Vaccine Evaluation Center (Sauvé, Scheifele), University of British Columbia, Vancouver, BC; Virology Section (Li), National Microbiology Laboratory, Winnipeg, Man.; Public Health Agency of Canada (Law), Ottawa, Ont.; Canadian Center for Vaccinology (Halperin), Dalhousie University, Halifax, NS; Public Health Agency of Canada/Canadian Institutes of Health Research Influenza Research Network (Rubinstein, Predy, Sauvé, Hammond, Aoki, Sikora, Li, Law, Halperin, Scheifele), Halifax, NS

Contributors: Each author contributed to the design of the study and to the development of the final manuscript. Ethan Rubinstein, Gerald Predy and Laura Sauvé were the site principal investigators; Greg Hammond, Fred Aoki, Chris Sikora and David Scheifele were site coinvestigators. Yan Li led the laboratory testing. David Scheifele was the project leader and oversaw analysis of the data. Scott Halperin and Barbara Law also contributed to the procurement of funding and administrative approvals. All of the authors approved the final version of the manuscript submitted for publication.

Funding: This study was entirely funded by a grant from the Canadian Institutes of Health Research and the Public Health Agency of Canada to the Influenza Research Network. The opinions expressed in this manuscript are solely those of the authors and neither necessarily reflect nor represent the position of the Public Health Agency of Canada.

Acknowledgements: The authors sincerely appreciate the diligent work of the research staff (nurses, coordinators, managers, statisticians and others) and the cooperation of the First Nations and Métis communities during a challenging time. This paper was presented in brief at the Canadian Immunization Conference, Québec City, Quebec, Dec. 3-5, 2010. 